\title{
1 Inference based PICRUSt accuracy varies across sample types and
}

\section{2 functional categories}

3 Shan Sun $^{1}$, Roshonda B. Jones ${ }^{2}$ and Anthony A. Fodor ${ }^{1}$

$4{ }^{1}$ Department of Bioinformatics and Genomics, University of North Carolina at Charlotte,

5 Charlotte, North Carolina, USA; ${ }^{2}$ The Saban Research Institute, Children's Hospital Los

6 Angeles, University of Southern California, Los Angeles, California, USA

\section{Abstract}

9 Background: Despite recent decreases in the cost of sequencing, shotgun metagenome

10 sequencing remains more expensive compared with 16S rRNA amplicon sequencing.

11 Methods have been developed to predict the functional profiles of microbial communities

12 based on their taxonomic composition, and PICRUSt is the most widely used of these

13 techniques. In this study, we evaluated the performance of PICRUSt by comparing the

14 significance of the differential abundance of functional gene profiles predicted with

15 PICRUSt to those from shotgun metagenome sequencing across different environments.

16 Results: We selected 7 datasets of human, non-human animal and environmental (soil)

17 samples that have publicly available $16 \mathrm{~S}$ rRNA and shotgun metagenome sequences. As

18 we would expect based on previous literature, strong Spearman correlations were

19 observed between gene compositions predicted with PICRUSt and measured with

20 shotgun metagenome sequencing. However, these strong correlations were preserved

21 even when the sample labels were shuffled. This suggests that simple correlation

22 coefficient is a highly unreliable measure for the performance of algorithms like

23 PICRUSt. As an alternative, we compared the performance of PICRUSt predicted genes 
24 to metagenome genes in inference models associated with metadata within each dataset.

25 With this method, we found reasonable performance for human datasets, with PICRUSt

26 performing better for inference on genes related to "house-keeping" functions. However,

27 the performance of PICRUSt degraded sharply outside of human datasets when used for

28 inference.

29 Conclusion: We conclude that the utility of PICRUSt for inference with the default

30 database is likely limited outside of human samples and that development of tools for

31 gene prediction specific to different non-human and environmental samples is warranted.

33 Key words: microbiota functional profile prediction, inference, sample type, functional

34 category

\section{Introduction}

37 Recent advances in next generation sequencing are revolutionizing our understanding of

38 complex microbial communities. Amplicon sequencing of marker genes provides

39 information regarding the phylogenetic diversity and taxonomic composition of

40 microorganisms present in the environment, while shotgun metagenome sequencing

41 provides additional information on the relative abundance of functional genes. Although

42 knowledge of taxonomy and functional genes of microorganisms are both important,

43 functional genes are more directly related to pathways and therefore are essential for

44 understanding the roles microorganisms play with regards to different physiological or

45 ecological outcomes. However, the higher cost of metagenome sequencing hinders its

46 application in studies consisting of a large number of samples, which are usually 
47 necessary in order to ensure adequate statistical power for detecting true differences [1].

48 Additionally, metagenome sequencing can also be very challenging for low biomass

49 samples or samples that are dominated by non-microbial DNA [2, 3].

51 In order to address this problem, tools have been developed to predict microbial

52 functional genes from their taxonomic compositions inferred from more cost-effective

53 amplicon sequencing, including PICRUSt, Tax4Fun and FaproTax [4-6]. Among these

54 tools, PICRUSt is the most widely used and has been applied in hundreds of projects on

55 various environments, including human gut [7, 8], murine [9, 10], fish [11], coral [12],

56 water [13], plant [14], bioreactor [15] and soil [16]. PICRUSt predicts the genes of

57 organisms without sequenced genomes based on mapping their 16S rRNA genes to

58 homologous taxa with fully sequenced genomes. The predictions of PICRUSt are

59 therefore limited by currently available genomes, which are highly biased towards

60 microorganisms associated with human health and biotechnology use [17].

62 In order to gauge the reliability of PICRUSt predictions in different environments and for

63 different functional categories, we utilized human, non-human animal (gorilla, mouse and

64 chicken) and environmental (soil) datasets that were sequenced for both 16S rRNA

65 marker genes and shotgun metagenomes. We compared the predicted functional profiles

66 from PICRUSt to the functional profiles measured with shotgun metagenome sequencing.

67 We demonstrated that simple correlations such as Spearman correlation overstate the

68 accuracy of PICRUSt by not taking into account the low variance of functional profiles

69 generated from shotgun metagenome sequencing. As an alternative metric, we used 
70 PICRUSt results for inference with simple statistical models and found reasonable

71 performance for human datasets, which presumably reflected the better reference

72 information we currently have for human genomes, but a sharp decrease in performance

73 for inference in non-human samples.

\section{Results}

76 Spearman correlation is not a reliable measurement for the prediction accuracy of gene

77 contents

78 In order to test the accuracy of PICRUSt from a range of environments, we compared

79 PICRUSt predictions to the results of shotgun metagenome sequencing on publicly

80 available datasets for which both metagenome and 16S rRNA sequences were available

81 (Table S1). As we would expect from previous literature [4], gene content estimation

82 from PICRUSt was robustly correlated with gene contents from metagenome sequencing

83 with Spearman correlations in the range of 0.62 to 0.84 (Fig. 1). For example, in one soil

84 sample (Fig. 1a), there is a clear correlation between the relative abundance of each gene

85 from PICRUSt and the relative abundance from metagenome sequencing (Spearman's

86 rho $=0.85)$. However, if the same comparison is made with the sample labels shuffled,

87 the correlation that was observed is not substantially impacted (Spearman's rho $=0.84$ )

88 (Fig. 1b).

89

90 The likely explanation for this observation is that across environments, there is less

91 variation between metagenome functional profiles of samples than their taxonomic

92 profiles (Fig. 2), an observation that has been previously made for human samples in the 
93 Human Microbiome Project [18]. In the datasets we examined, despite sample labels

94 being shuffled, the relative abundance of genes from PICRUSt estimates were highly

95 correlated with the relative abundance of gene estimates from metagenome sequencing,

96 with correlation coefficients always higher than 0.5. The PICRUSt predictions were often

97 only marginally higher on the unpermuted data than those with sample labels shuffled,

98 with perhaps the gorilla dataset as an exception (Fig. 1c). However, even in the gorilla

99 samples, the difference between Spearman coefficients for permuted and unpermuted

100 samples was only 0.12 . For the 2 soil datasets, the Spearman coefficients for the

101 unpermuted samples were not significantly different from those for the permuted samples

$102 \quad(\mathrm{P}=0.508$ and 0.794$)$.

103

104 PICRUSt results for inference showed higher consistency with metagenome sequences in

\section{5 human samples than non-human samples}

106 As an alternative evaluation to Spearman correlation, we compared how PICRUSt's gene

107 predictions performed in a t-test to genes detected with shotgun metagenome sequencing

108 in each of our datasets. For this purpose, we formed a null hypothesis for each gene in

109 each dataset that there is no difference in the mean of that gene's distribution of relative

110 abundance between the two groups in the dataset. For example, for each of the 5,574

111 genes detected by both PICRUSt and metagenome sequencing in the Human_KW dataset,

112 we used a t-test to generate a P-value for the difference in gene composition between

113 rural and urban samples. Across all the genes, there was a reasonable correlation

$114(\mathrm{rho}=0.49)$ of P-values from t-tests run on metagenome sequencing data and those on

115 PICRUSt data (Fig. 3; top left panel). Unlike our results for Spearman correlation, this 
116 rho value is sensitive to sample permutation, as when we repeated this procedure on

117 permuted data, the correlation between P-values generated from metagenome sequencing

118 and those from PICRUSt approached zero (Fig. 4). We saw a similarly robust correlation

119 (rho=0.554) for our Human_TY dataset evaluating a null hypothesis comparing US and

120 non-US samples. However, when we extended this analysis to non-human datasets (using

121 the null hypotheses for each study listed in Table S1), the inference produced by

122 PICRUSt showed a much lower similarity to inference produced by metagenome

123 sequencing (Fig. 3).

125 In order to ensure that the differences in performance were not due to differences in

126 sample sizes, we randomly sub-sampled each larger dataset (without replacement) to 10

127 samples (5 per group) and re-calculated the comparison of P-values between PICRUSt

128 and metagenome sequencing (Fig. S1). Even at a smaller size, data from the human

129 studies showed greater concordance than those from other environments. We conclude

130 that the difference in sample sizes between datasets does not explain the variability of

131 PICRUSt accuracy between different samples types in our study. Likewise, the effect

132 sizes of the associations with metadata, measured as $\mathrm{R}^{2}$ in a PERMANOVA test, were

133 not substantially higher in human samples (Table S1). It therefore also seems unlikely

134 that effect size alone can explain the better concordance we observed between inference

135 results from PICRUSt and metagenome sequencing for human samples.

137 In order to further investigate the consistency of PICRUSt and metagenome sequencing,

138 we next asked how many genes were detected by both methods or by each method 
139 individually. For some datasets, such as the Human_KW dataset, PICRUSt failed to

140 predict many genes that were detected by metagenome sequencing. For other datasets,

141 such as the soil datasets, many genes that PICRUSt predicted were not detected in

142 metagenome sequencing and there were also many genes seen in metagenome

143 sequencing but not in PICRUSt (Fig. 3). For the chicken dataset with an average

144 metagenome sequencing depth of 31 million reads/sample and the gorilla dataset of 27

145 million reads/sample, $23.4 \%$ and $26.1 \%$ of PICRUSt predicted genes could not be

146 detected by metagenome sequencing (Fig. 3). In addition, the metagenome sequencing of

147 the Human_KW dataset with an average sequencing depth of 10 million reads/sample

148 detected 13,880 genes and PICRUSt missed 59.8\% of them (Fig. 3).

150 PICRUSt performs differently for different functional categories

151 We next investigated the discrepancy between PICRUSt and metagenome sequencing for

152 inference in different functional categories (Fig. 5). When comparing the P-values from

153 PICRUSt data to P-values from metagenome sequencing, there was a lack of significant

154 correlations in the human gut samples for some categories including Signaling molecules

155 and interaction, Immune system, Endocrine system, Cell growth and death, Nervous

156 system, Digestive system, Environmental adaptation, Cardiovascular diseases, Immune

157 diseases, Substance dependence, Circulatory system, Excretory system and Viral protein

158 family. PICRUSt appeared to have a closer match to the metagenome sequencing data for

159 categories such as Folding, sorting and degradation, Translation, Transcription,

160 Replication and repair, Nucleotide metabolism, Glycan biosynthesis and metabolism,

161 Drug resistance, Neurodegenerative diseases, Endocrine and metabolic diseases and 
162 Aging. For the genes only detected by one method, most of the genes missed by

163 PICRUSt belong to Environmental Information Processing, Organismal Systems and

164 Human Diseases, while those associated with Metabolism were more likely predicted

165 (Fig. S2). Among the genes predicted by PICRUSt but not detected by metagenome

166 sequencing, most of them belong to Cellular processing and signaling, Signaling

167 molecules and interaction, Genetic information processing, Substance Dependence and

168 Viral protein family (Fig. S3).

170 Discussion

171 Microbial community functional profiles are typically of much lower variance compared

172 to their taxonomic profiles likely because of the large proportions of "core" or

173 "housekeeping" functions [18-20]. In this study, we showed that this lack of variance in

174 functional profiles between samples leads to a strong correlation between functional

175 profiles from metagenome sequencing and those estimated from references with

176 PICRUSt, even when the sample labels are randomly shuffled (Fig. 1c). We argue that

177 this result shows that metrics commonly used to measure gene prediction performance,

178 such as Spearman correlation between gene composition estimated with PICRUSt and

179 metagenome sequencing, do not give a satisfactory measure of overall accuracy. As an

180 alternative, we evaluated the performance of PICRUSt at a community level based on

181 inference from simple statistical models testing the association between genes and

182 metadata. Unlike simple Spearman correlations, evaluation with inference methods are

183 highly sensitive to shuffling sample labels (Fig. 4), which indicated that inference

184 methods are much less affected by the relatively low variance of functional profiles. The 
185 inference-based approach also has the advantage of reflecting the common use of

186 PICRUSt to reveal predicted functional profiles associated with different metadata

187 categories $[11,12,21-23]$. Incorrect estimation of differential abundance could lead to

188 false discovery of signature genes, and this concern motivated our approach to determine

189 the reliability of PICRUSt produced inference in different systems.

191 In this study, we selected 7 datasets from different environments which include human,

192 non-human animal and environmental (soil) samples. With inference methods, we found

193 that PICRUSt and metagenome sequencing had more consistent assessment from human

194 datasets than non-human animals or environmental datasets. It is likely that these

195 differences reflected the bias of genome databases towards human-related

196 microorganisms. However, PICRUSt still missed a large percentage of genes that were

197 detected with metagenome sequencing in human samples, and an increase in metagenome

198 sequencing depth could presumably increase the number of genes that are potentially not

199 detected by PICRUSt (Fig. 3; Table S1). Likewise, PICRUSt sometimes predicted many

200 genes not found in metagenome sequencing even in samples with presumably adequate

201 sequencing depth of millions of reads per sample, which suggested that these additional

202 genes are likely false predictions.

203

204 As a meta-analysis across multiple studies, there are systemic factors that may influence

205 the results of this study, including different sample sizes, sequencing designs and effect

206 sizes of associations with the metadata. We repeated our analysis on subsampled datasets

207 that were rarified to the number of samples in the smallest dataset that we examined and 
208 observed a similar pattern of results with inference more consistent between PICRUSt

209 and metagenome sequencing for human studies (Fig. S1). This result suggests that

210 difference in sample size does not explain the better inference performance of PICRUSt

211 for the human studies. While differences in effect size and experimental design are harder

212 to control, the human studies did not have an obviously higher effect size than the non-

213 human studies as measured with a PERMANOVA test (Table S1). It therefore also seems

214 unlikely that differences in effect sizes of associations with the metadata can explain our

215 results.

217 Our study also examined the performance of PICRUSt for different functional categories.

218 This approach was motivated by the presumed bias in current genome databases toward

219 culturable microorganisms [24]. We reasoned that the unculturable state of

220 microorganisms could be caused by specific requirements for nutrients, temperature, $\mathrm{pH}$,

221 beneficial interactions with other microbes or extremely slow growth rates [25], which in

222 turn could lead to bias in gene families in different microorganisms. Likewise, different

223 microorganisms and genes also have different rates of horizontal gene transfer and the

224 accuracy of gene content estimation may therefore vary depending on the type of the

225 genes and microbial groups [26]. We found that PICRUSt performed best for "house-

226 keeping" functions such as transcription and translation while the accuracy of functions

227 related to environmental information processing was generally much lower (Fig. 5).

228 Future algorithms for gene prediction could explicitly incorporate this performance

229 variance into a confidence score that could give users estimated error rates for prediction

230 of a given gene family. 
232 Our analysis suggests that in order to better predict microbial functional profiles in

233 certain environments, it will be of utility to develop tools specific to that environment.

234 There have been encouraging examples in the literature of efforts to make environmental

235 specific databases such as CowPI, a functional inference tool specific to the rumen

236 microbiome, which had better estimates than PICRUSt when used for predicting

237 functional profiles in the bovine environment [27]. We can look forward to similar future

238 refinements in the next generation of these algorithms that will use appropriate reference

239 databases for an environment and analyze individual functional categories to yield

240 confidence scores for each prediction.

\section{Methods}

243 The datasets used in this study include 2 human datasets (named as Human_KW [28] and

244 Human_TY [29] in our study after the initials of their first authors), 1 gorilla [30], 1

245 mouse [31], 1 chicken [32] and 2 soil datasets Soil_LWM [33] and Soil_AAN [34]. Each

246 dataset has publicly available 16S rRNA and metagenome sequences and is associated

247 with a two-level categorical metadata. The Human_KW study compared urban and rural

248 subjects in China, while US and non-US subjects were compared for the Human_TY

249 study. In the gorilla study, the dry and wet seasons were compared while the mouse study

250 compared community composition of two enterotypes. Lean and fat broiler chicken lines

251 were compared for the chicken study. For the Soil_LWM study, Amazon dark earth and

252 agricultural soil were compared, while forested and deforested soils were compared for

253 the Soil_AAN study. Information regarding data locations, sequencing depth, sample 
254 sizes and effect sizes (measured as $\mathrm{R}^{2}$ in the PERMANOVA test with the function

255 'adonis' in the R package 'vegan') for each study are listed in Table S1.

257 The PICRUSt predictions of the 16S rRNA sequences in the datasets followed the

258 developer's instructions [4]. The authors' metagenome analysis results were used when

259 available $[29,31,33,34]$, otherwise the raw sequences were analyzed with humann2

260 following the developer's instructions [35]. In each dataset, all PICRUSt-predicted gene

261 families and pathways were compared to those from metagenome sequencing to

262 determine their discrepancy in the number and types of genes revealed. For genes

263 detected by both PICRUSt and metagenome sequencing, we used two sets of methods to

264 evaluate their consistency. In a first set of methods, we analyzed the Spearman

265 correlation between PICRUSt-predicted gene composition and those from metagenome

266 sequencing. As a control, we permuted the sample labels 100 times and re-calculated

267 Spearman correlation between PICRUSt estimates and metagenome sequencing estimates

268 with sample labels shuffled.

270 In a second set of methods, we analyzed the consistency of PICRUSt and metagenome

271 sequencing in the P-values they generated for null hypotheses of no association with

272 metadata. For this purpose, P-values were produced with a t-test of the 2 distinguishable

273 groups in each dataset (Table S1). P-values from the t-test were log10 transformed and

274 multiplied by either 1 or -1 to include the direction of change as indicated below:

$$
\mathrm{P} \_\mathrm{t}=\log 10(\mathrm{P})^{*} \mathrm{t} / \mathrm{abs}(\mathrm{t})
$$


$276 \quad \mathrm{P} \_\mathrm{t}$ is the transformed $\mathrm{P}$-value, $\mathrm{P}$ is the $\mathrm{P}$-value from $\mathrm{t}$ test, and $\mathrm{t}$ is the statistic of $\mathrm{t}$-test.

277 We then estimated the consistency of the log-transformed P-values from PICRUSt and

278 metagenome sequencing with Spearman's correlation. To determine whether this method

279 is affected by the low variance of functional profiles, we permuted sample labels of the

280 metagenome sequencing gene compositions 100 times and re-calculated the log-

281 transformed P-values and their correlation with the PICRUSt results.

283 In order to correct for differences in sample size, each dataset was also subsampled to 5

284 samples per group to ensure that the different sample sizes of datasets were not unduly

285 influencing our results. The PICRUSt predictions and metagenome sequencing were also

286 compared in each of the 48 level 2 functional categories.

\section{Declarations}

\section{Availability of data and material}

290 The datasets analyzed in this study are publicly available with repositories and accession

291 numbers listed in Table S1. R scripts used in this study are available at Github

292 (https://github.com/ssun6/Inference_picrust). Additional requests and questions can be

293 addressed to S.S.

\section{Competing interests}

295 The authors declare that they have no competing interests. 


\section{References}

$300 \quad$ 1. Button KS, Ioannidis JP, Mokrysz C, Nosek BA, Flint J, Robinson ES, Munafò

$301 \quad$ MR: Power failure: why small sample size undermines the reliability of

302

303 neuroscience. Nature Reviews Neuroscience 2013, 14:365.

304

2. Jervis-Bardy J, Leong LE, Marri S, Smith RJ, Choo JM, Smith-Vaughan HC, Nosworthy E, Morris PS, O'Leary S, Rogers GB: Deriving accurate microbiota profiles from human samples with low bacterial content through post-

sequencing processing of Illumina MiSeq data. Microbiome 2015, 3:19.

3. Minich JJ, Zhu Q Janssen S, Hendrickson R, Amir A, Vetter R, Hyde J, Doty MM, Stillwell K, Benardini J: KatharoSeq enables high-throughput microbiome analysis from low-biomass samples. MSystems 2018, 3:e00218-00217.

4. Langille MGI, Zaneveld J, Caporaso JG, McDonald D, Knights D, Reyes JA, Clemente JC, Burkepile DE, Vega Thurber RL, Knight R, et al: Predictive functional profiling of microbial communities using $16 \mathrm{~S}$ rRNA marker gene sequences. Nat Biotech 2013, 31:814-821.

5. Aßhauer KP, Wemheuer B, Daniel R, Meinicke P: Tax4Fun: predicting functional profiles from metagenomic 16 S rRNA data. Bioinformatics 2015, 31:2882-2884.

6. Louca S, Parfrey LW, Doebeli M: Decoupling function and taxonomy in the global ocean microbiome. Science 2016, 353:1272-1277.

7. Gevers D, Kugathasan S, Denson LA, Vázquez-Baeza Y, Van Treuren W, Ren B, Schwager E, Knights D, Song SJ, Yassour M: The treatment-naive microbiome in new-onset Crohn's disease. Cell host \& microbe 2014, 15:382-392.

8. Goodrich JK, Waters JL, Poole AC, Sutter JL, Koren O, Blekhman R, Beaumont M, Van Treuren W, Knight R, Bell JT: Human genetics shape the gut microbiome. Cell 2014, 159:789-799.

9. Carmody RN, Gerber GK, Luevano Jr JM, Gatti DM, Somes L, Svenson KL, Turnbaugh PJ: Diet dominates host genotype in shaping the murine gut microbiota. Cell host \& microbe 2015, 17:72-84.

10. De Palma G, Blennerhassett P, Lu J, Deng Y, Park A, Green W, Denou E, Silva M, Santacruz A, Sanz Y: Microbiota and host determinants of behavioural phenotype in maternally separated mice. Nature communications 2015, 6:7735.

11. Bolnick DI, Snowberg LK, Hirsch PE, Lauber CL, Org E, Parks B, Lusis AJ, Knight R, Caporaso JG, Svanbäck R: Individual diet has sex-dependent effects on vertebrate gut microbiota. Nature communications 2014, 5:ncomms5500.

12. Ainsworth TD, Krause L, Bridge T, Torda G, Raina J-B, Zakrzewski M, Gates RD, Padilla-Gamiño JL, Spalding HL, Smith C: The coral core microbiome identifies rare bacterial taxa as ubiquitous endosymbionts. The ISME journal 2015, 9:2261.

13. Revetta R, Gomez-Alvarez V, Gerke T, Santo Domingo J, Ashbolt N: Changes in bacterial composition of biofilm in a metropolitan drinking water 
342 14. Zarraonaindia I, Owens SM, Weisenhorn P, West K, Hampton-Marcell J, Lax S, Bokulich NA, Mills DA, Martin G, Taghavi S: The soil microbiome influences grapevine-associated microbiota. MBio 2015, 6:e02527-02514.

15. Li X, Sun S, Yuan H, Badgley BD, He Z: Mainstream upflow nitritationanammox system with hybrid anaerobic pretreatment: Long-term performance and microbial community dynamics. Water research 2017, 125:298-308.

16. Ling N, Zhu C, Xue C, Chen H, Duan Y, Peng C, Guo S, Shen Q: Insight into how organic amendments can shape the soil microbiome in long-term field experiments as revealed by network analysis. Soil Biology and Biochemistry 2016, 99:137-149.

17. Choi J, Yang F, Stepanauskas R, Cardenas E, Garoutte A, Williams R, Flater J, Tiedje JM, Hofmockel KS, Gelder B: Strategies to improve reference databases for soil microbiomes. The ISME journal 2017, 11:829.

18. Huttenhower C, Gevers D, Knight R, Abubucker S, Badger JH, Chinwalla AT, Creasy HH, Earl AM, FitzGerald MG, Fulton RS: Structure, function and diversity of the healthy human microbiome. nature 2012, 486:207.

19. Franzosa EA, Hsu T, Sirota-Madi A, Shafquat A, Abu-Ali G, Morgan XC, Huttenhower C: Sequencing and beyond: integrating molecular'omics' for microbial community profiling. Nature Reviews Microbiology 2015, 13:360.

20. Ortiz-Álvarez R, Fierer N, de los Ríos A, Casamayor E0, Barberán A: Consistent changes in the taxonomic structure and functional attributes of bacterial communities during primary succession. The ISME journal 2018, 12:1658.

21. David LA, Maurice CF, Carmody RN, Gootenberg DB, Button JE, Wolfe BE, Ling AV, Devlin AS, Varma Y, Fischbach MA: Diet rapidly and reproducibly alters the human gut microbiome. Nature 2014, 505:559.

22. Mao S, Zhang M, Liu J, Zhu W: Characterising the bacterial microbiota across the gastrointestinal tracts of dairy cattle: membership and potential function. Scientific reports 2015, 5:16116.

23. Loudon AH, Woodhams DC, Parfrey LW, Archer H, Knight R, McKenzie V, Harris RN: Microbial community dynamics and effect of environmental microbial reservoirs on red-backed salamanders (Plethodon cinereus). The ISME journal 2014, 8:830.

24. Grice EA, Segre JA: The human microbiome: our second genome. Annual review of genomics and human genetics 2012, 13:151-170.

25. Vartoukian SR, Palmer RM, Wade WG: Strategies for culture of 'unculturable'bacteria. FEMS microbiology letters 2010, 309:1-7.

26. Gogarten JP, Townsend JP: Horizontal gene transfer, genome innovation and evolution. Nature Reviews Microbiology 2005, 3:679.

27. Wilkinson TJ, Huws SA, Edwards JE, Kingston-Smith AH, Siu-Ting K, Hughes M, Rubino F, Friedersdorff M, Creevey CJ: CowPI: A Rumen Microbiome Focussed Version of the PICRUSt Functional Inference Software. Front Microbiol 2018, 9:1095.

28. Winglee K, Howard AG, Sha W, Gharaibeh RZ, Liu J, Jin D, Fodor AA, GordonLarsen P: Recent urbanization in China is correlated with a Westernized 
microbiome encoding increased virulence and antibiotic resistance genes. Microbiome 2017, 5:121.

29. Yatsunenko T, Rey FE, Manary MJ, Trehan I, Dominguez-Bello MG, Contreras M, Magris M, Hidalgo G, Baldassano RN, Anokhin AP: Human gut microbiome viewed across age and geography. nature 2012, 486:222.

30. Hicks AL, Lee KJ, Couto-Rodriguez M, Patel J, Sinha R, Guo C, Olson SH, Seimon A, Seimon TA, Ondzie AU: Gut microbiomes of wild great apes fluctuate seasonally in response to diet. Nature communications 2018, 9:1786.

31. Wang J, Linnenbrink M, Künzel S, Fernandes R, Nadeau M-J, Rosenstiel P, Baines JF: Dietary history contributes to enterotype-like clustering and functional metagenomic content in the intestinal microbiome of wild mice. Proceedings of the National Academy of Sciences 2014:201402342.

32. Hou Q, Kwok L-Y, Zheng Y, Wang L, Guo Z, Zhang J, Huang W, Wang Y, Leng L, Li H: Differential fecal microbiota are retained in broiler chicken lines divergently selected for fatness traits. Scientific reports 2016, 6:37376.

33. Mendes LW, Raaijmakers JM, de Hollander M, Mendes R, Tsai SM: Influence of resistance breeding in common bean on rhizosphere microbiome composition and function. The ISME journal 2017, 12:212.

34. Navarrete AA, Tsai SM, Mendes LW, Faust K, de Hollander M, Cassman NA, Raes J, van Veen JA, Kuramae EE: Soil microbiome responses to the short-term effects of Amazonian deforestation. Molecular ecology 2015, 24:2433-2448.

35. Franzosa EA, McIver LJ, Rahnavard G, Thompson LR, Schirmer M, Weingart G, Lipson KS, Knight R, Caporaso JG, Segata N: Species-level functional profiling of metagenomes and metatranscriptomes. Nature methods 2018, 15:962.

414 Fig. 1. Spearman correlations between PICRUSt and shotgun metagenome sequencing in

415 unpermuted and permuted datasets. A and B: An example showing the correlations

416 between genes relative abundances estimated by PICRUSt and metagenome sequencing

417 in a soil sample (sample BulkAG3 in soil_AAN dataset) for unpermuted (A) and

418 permuted (B) sample labels. C: Comparison of Spearman's rho between PICRUSt and

419 metagenome sequencing in unpermuted (red) and permuted data (blue) in all datasets. In

420 each of the 100 permutations, all sample labels were shuffled for every gene. 
422 Fig. 2. Taxonomic (A) and functional profiles (B) of the 7 datasets in our study. The

423 taxonomic profiles were plotted at the class level and the functional profiles were plotted

424 at the broadest functional category of the KEGG database for visualization.

425

426 Fig. 3. Comparison of inferences based on gene composition estimated with PICRUSt

427 and metagenome sequencing in each of the 7 datasets. The Venn diagrams (left in each

428 panel) show the number of genes detected by both PICRUSt and metagenome sequencing

429 or by only one method, with those detected by PICRUSt in pink and those by

430 metagenome sequencing in blue. The plots (right in each panel) show log-transformed P-

431 values of the t-test evaluating the null hypothesis for each dataset (see methods and Table

432 S1) from metagenome sequencing (x-axis) and PICRUSt (y-axis) for genes in common

433 between the two methods. The sign of the log-transformed P-values reflect the direction

434 of change (see methods). For example, in the Human_KW dataset, genes higher in urban

435 subjects are in the upper-right hand quadrant and genes lower in urban are in the lower-

436 right hand quadrant. The table (inset lower right corner) shows the percentage of genes

437 called by only one method for each dataset and the coefficients of Spearman's

438 correlations between log-transformed P-values based on gene composition estimated by

439 PICRUSt and metagenome sequencing.

441 Fig. 4. The results of inference methods in unpermuted and permuted datasets. The red

442 points are the Spearman's rho of log-transformed P-values from PICRUSt and

443 unpermuted metagenome sequencing data for each dataset. The boxplots of blue points

444 show the Spearman's rho of log-tranformed P-values from PICRUSt and permuted 
445 metagenome sequencing data in 100 permutations. In each of the 100 permutations, all

446 sample labels were shuffled for every gene.

447

448 Fig. 5. Spearman's rho of the correlations between log-transformed P-values from

449 PICRUSt and metagenome sequencing in 48 KEGG functional categories at the second

450 hierarchy level with the bar colors indicating the functional categories at the first

451 hierarchy level. 


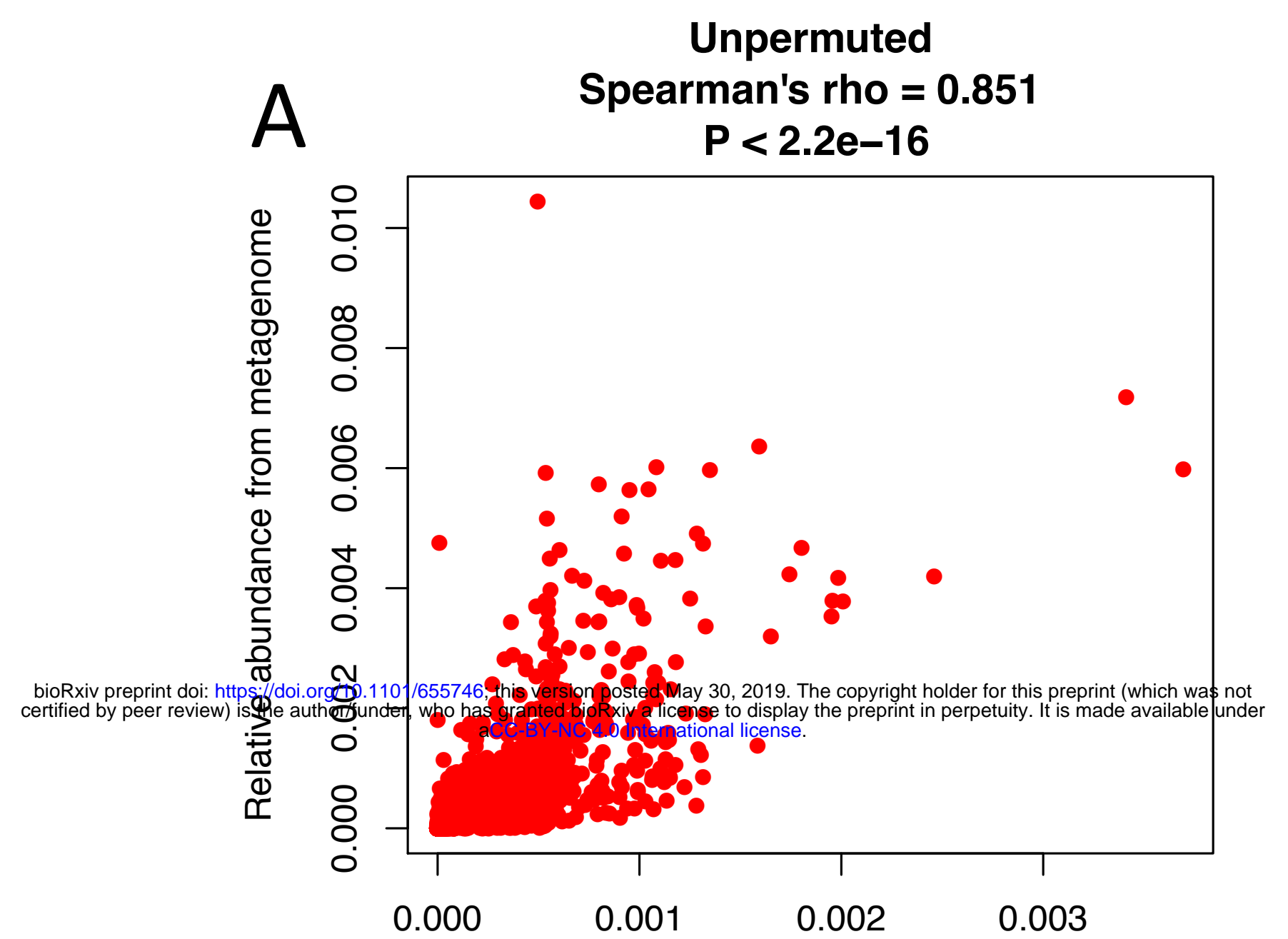

Relative abundance from PICRUSt

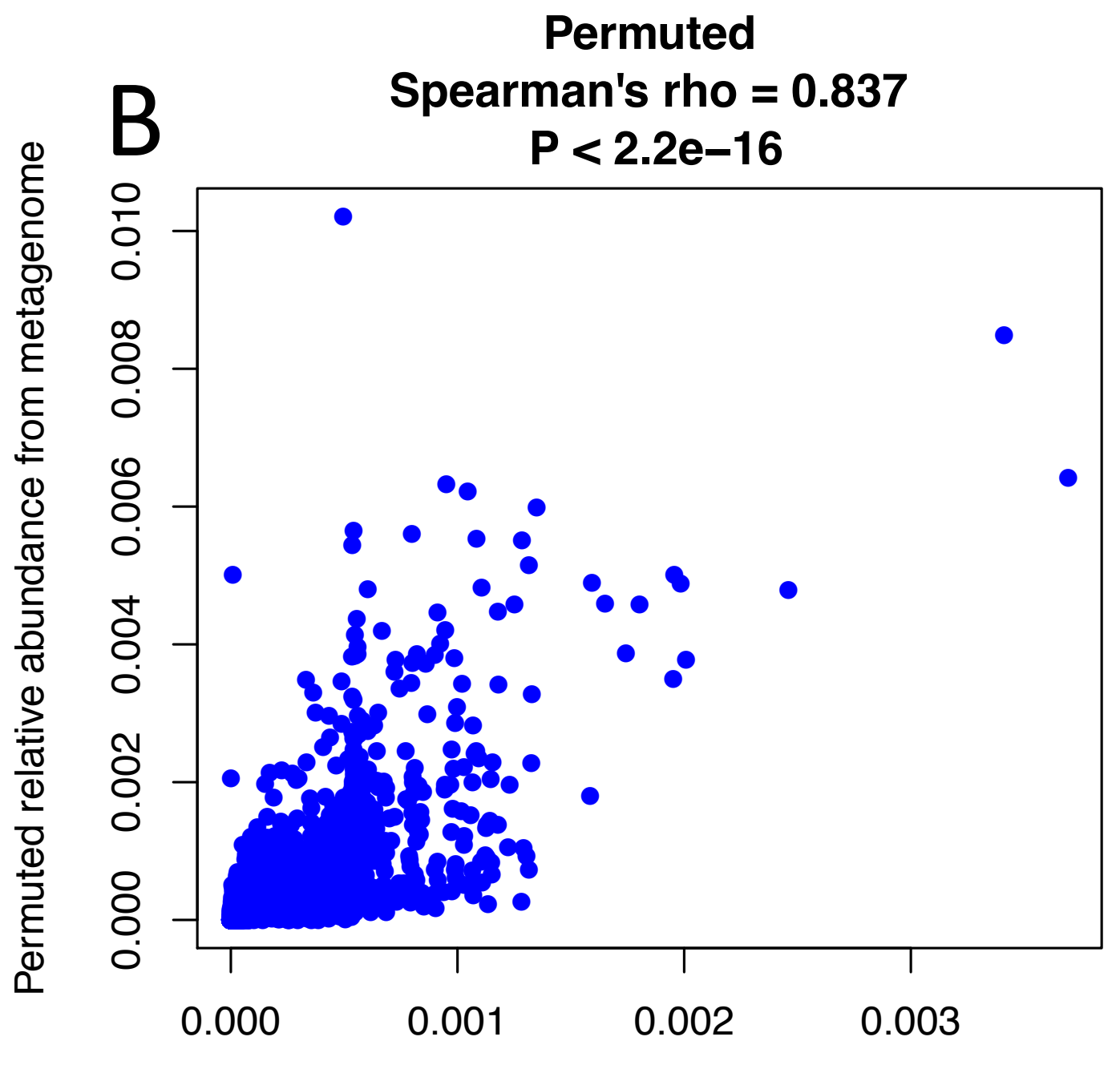

Relative abundance from PICRUSt

C

Mean of the Spearman's rho (unpermuted)

Mean of the Spearman's rho (permuted)

$P$-value of the difference between the 2 sets of rho

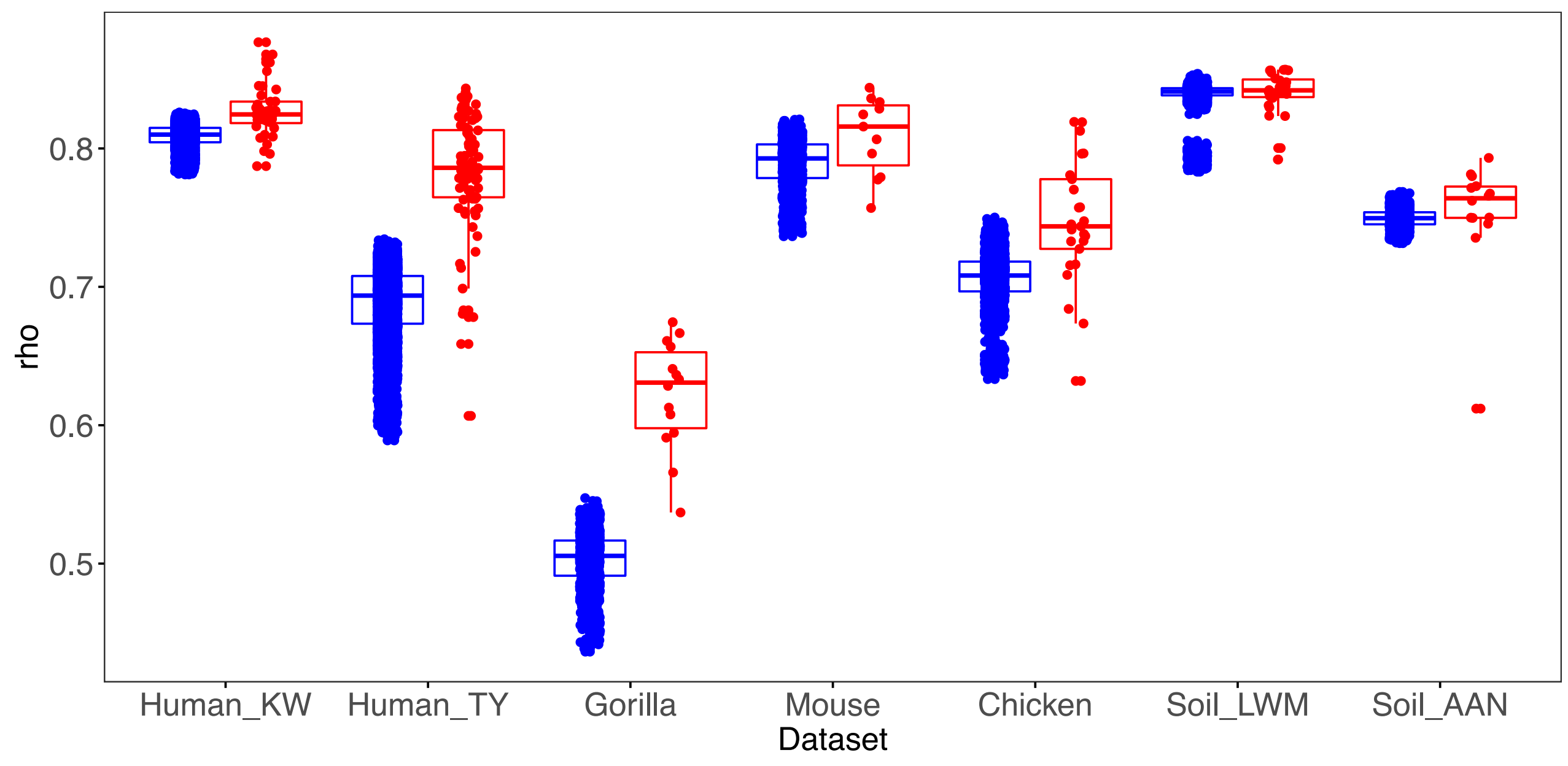

Group

的 permuted

审 unpermuted
0.827
$\begin{array}{lll}0.782 & 0.622 \quad 0.809\end{array}$
$0.747 \quad 0.840$
0.753
0.809
0.688
0.503
0.789
0.706
0.838
0.750

$4.41 \mathrm{E}-07<2.2 \mathrm{e}-16$

4.6E-08

0.0435

0.000142

0.505

0.794 


\section{Human_KW}

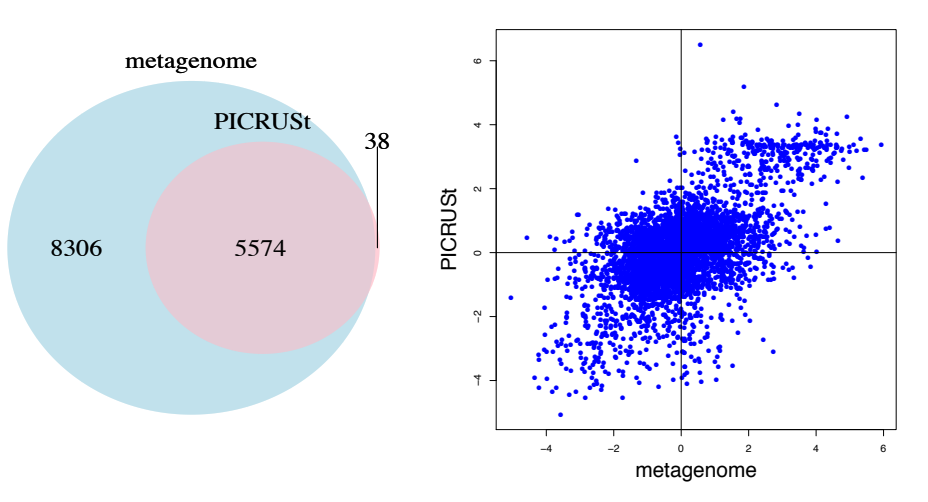

Mouse

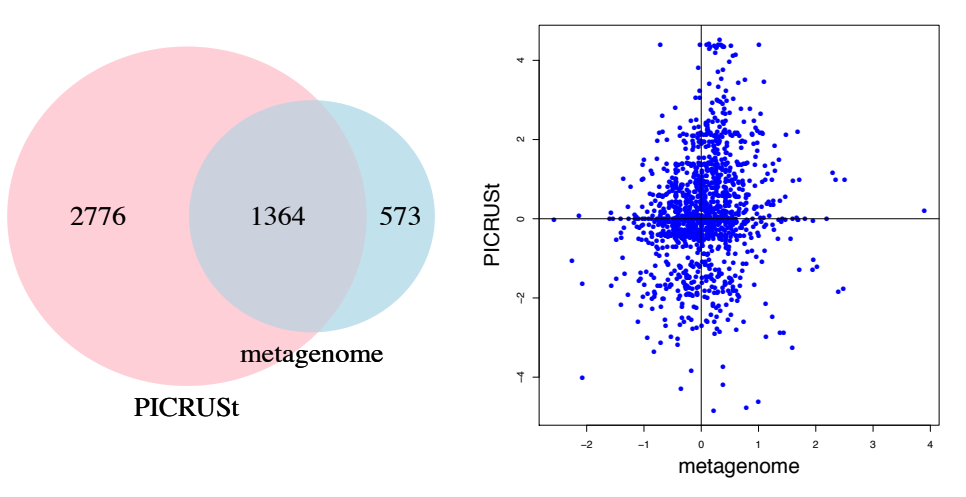

Soil_LWM

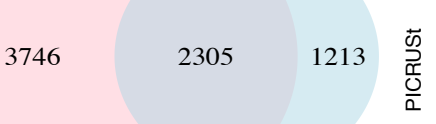

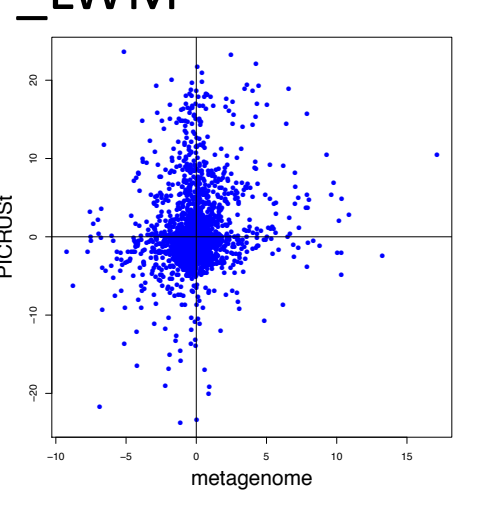

\section{Human_TY}

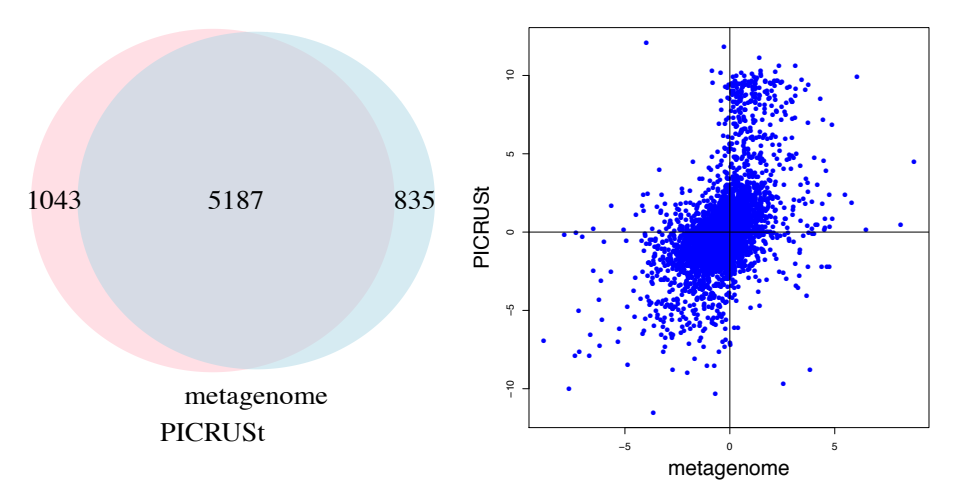

Chicken
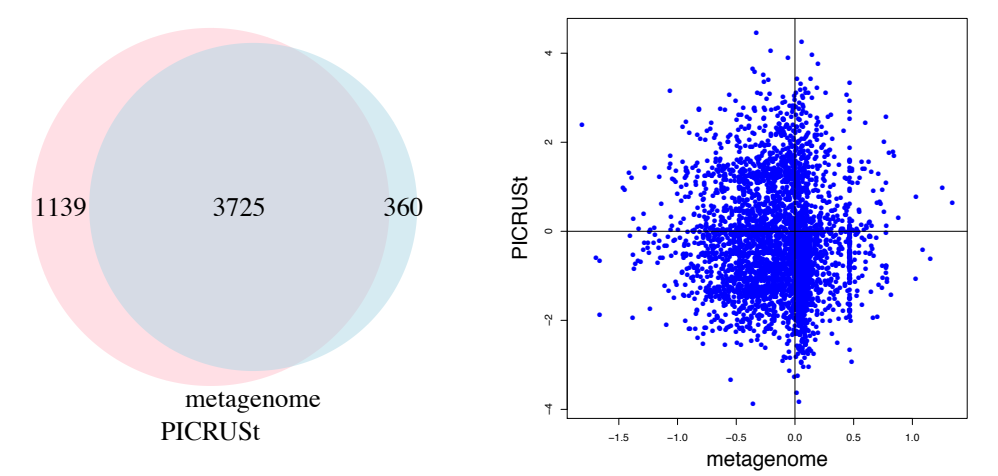

Soil_AAN
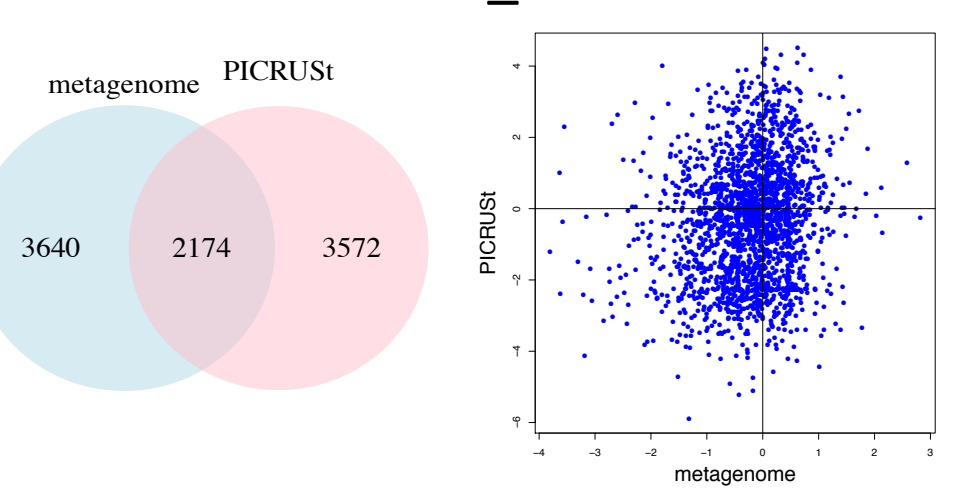

\section{Gorilla}
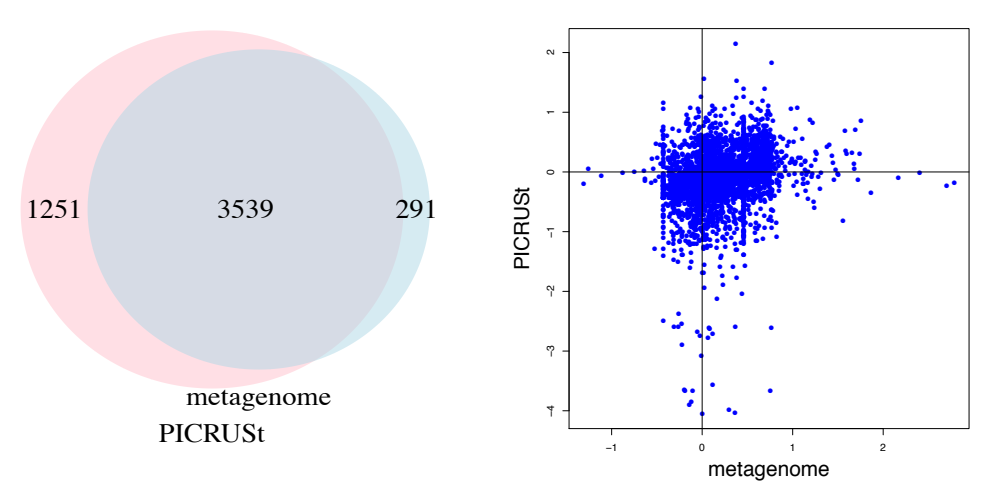

PICRUSt

metagenome

\begin{tabular}{llll}
\hline Dataset & $\begin{array}{l}\text { Percentage of } \\
\text { genes not } \\
\text { predicted by } \\
\text { PICRUSt }\end{array}$ & $\begin{array}{l}\text { Percentage of } \\
\text { genes predicted } \\
\text { but not in } \\
\text { metagenome }\end{array}$ & $\begin{array}{l}\text { Spearman's } \\
\text { rho of } \\
\text { transformed } \\
\text { P-values }\end{array}$ \\
\hline Human_KW & $59.8 \%$ & $0.68 \%$ & $0.490^{*}$ \\
Human_TY & $13.9 \%$ & $16.7 \%$ & $0.554^{*}$ \\
Gorilla & $7.60 \%$ & $26.1 \%$ & $0.313^{*}$ \\
Mouse & $29.6 \%$ & $67.1 \%$ & $0.206^{*}$ \\
Chicken & $8.81 \%$ & $23.4 \%$ & -0.0324 \\
Soil_LWM & $34.5 \%$ & $61.9 \%$ & $0.125^{*}$ \\
Soil_AAN & $62.6 \%$ & $62.2 \%$ & $0.127^{*}$ \\
\hline
\end{tabular}




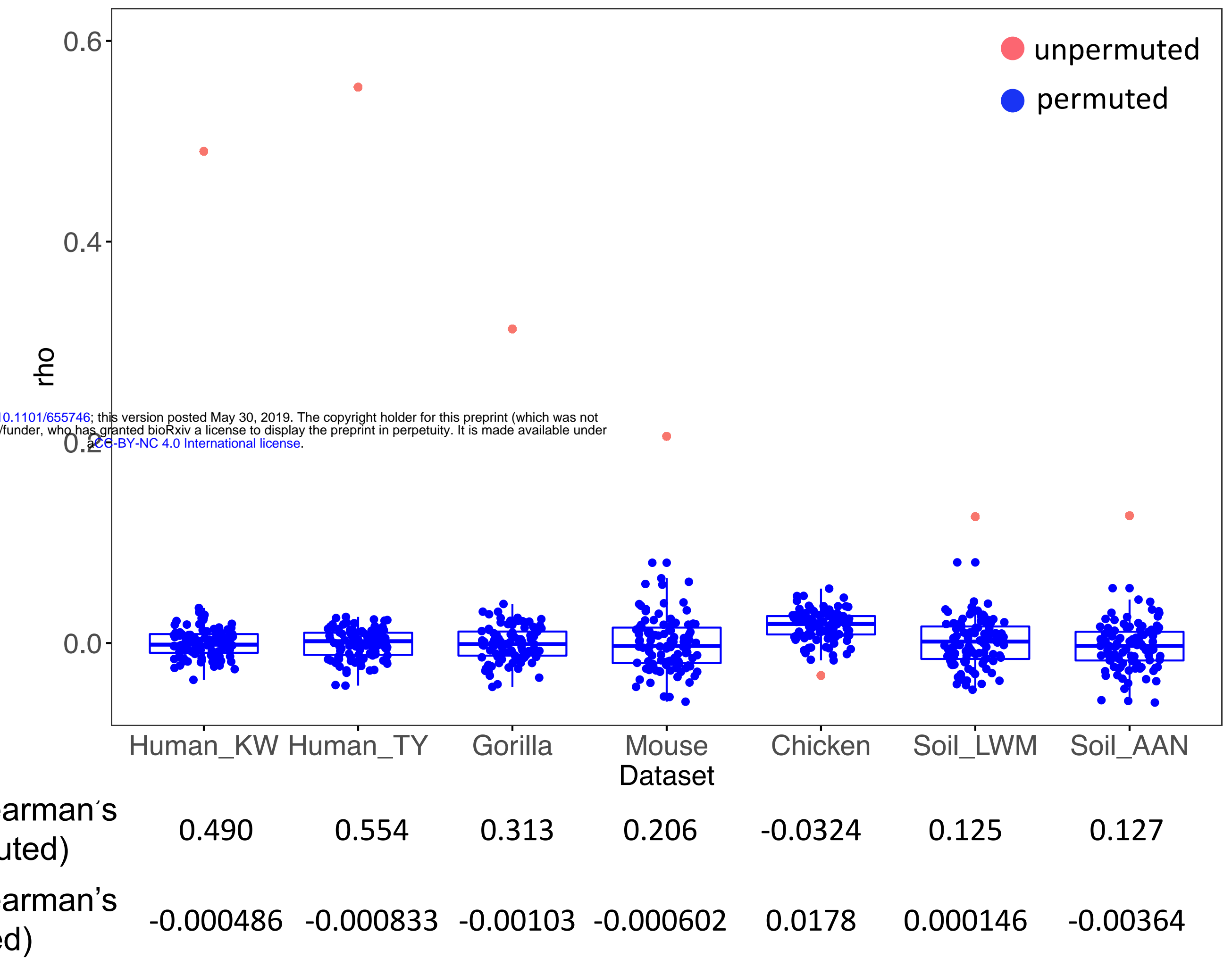




\section{Human_KW}

09132 Signal transduction

09133 Signaling molecules and interaction

09123 Folding, sorting and degradation

09122 Translation

09112 Enzyme families
09121 Transcription

09141 Transport and catabolism

09151 Immune system
09191 Metabolism

09152 Endocrine system

09107 Glycan biosynthesis and metabolism

09109 Metabolism of terpenoids and polyketides

09102 Energy metabolism

09156 Nervous system 09142 Cell motility

09111 Xenobiotics biodegradation and metabolism

Biosynthesis of other secondary metabolites
09193 Cellular processes and signaling

09145 Cellor 09 Poorly characterized

09163 Neurodegenerative diseases
09166 Endocrine and metabolic diseases

09144 Cellular community - eukaryotes

09104 Nucleotide metabolism
09154 Digestive system

09159 Environmental adaptation

09125 RNA family
0

09162 Immune diseases
09164 Substance dependence

09153 Circulatory system

09158 Development

09192 Genetic information processing
09106 Metabolism of other amino acids

09157 Sensory system

09155 Excretory system
09196 Viral protein family
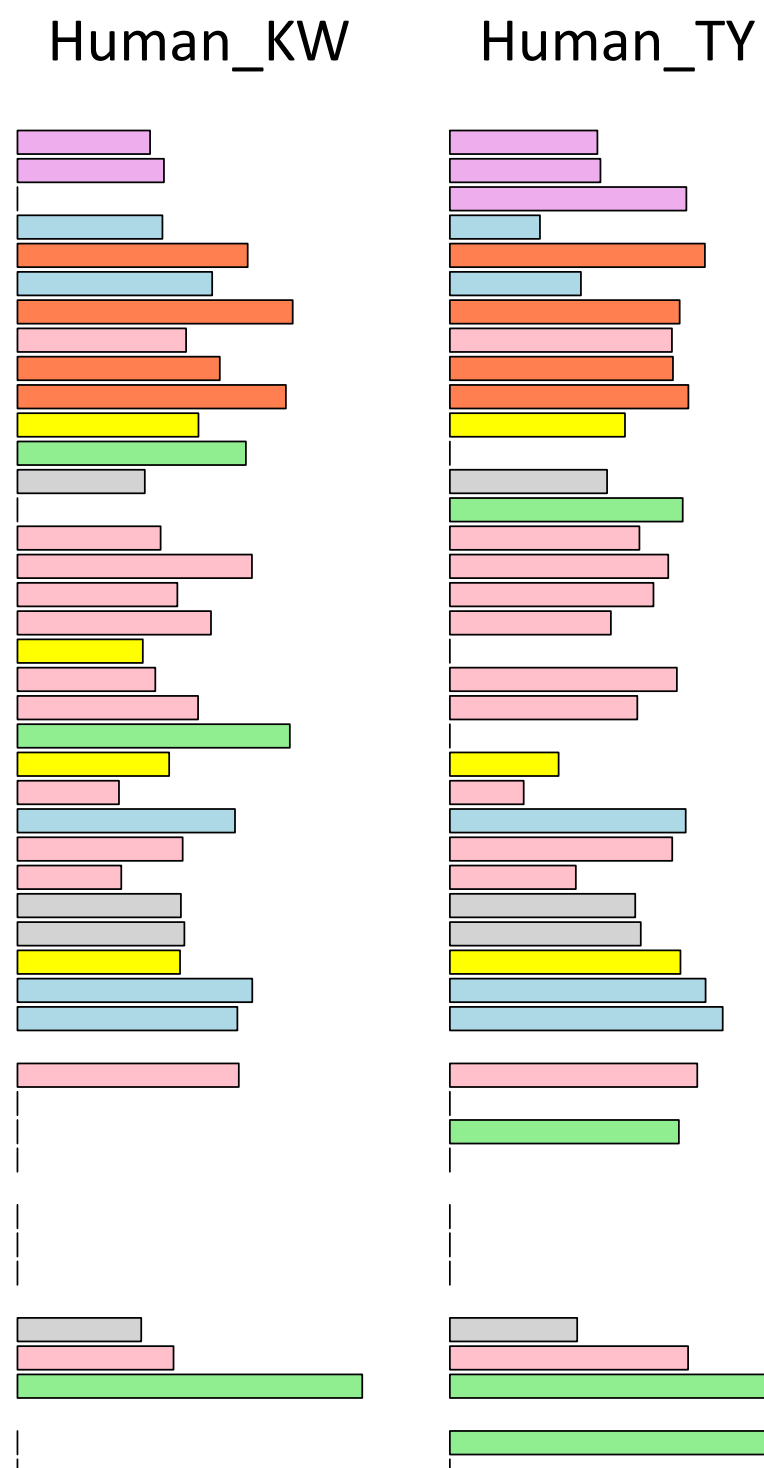

Gorilla

Mouse

Chicken
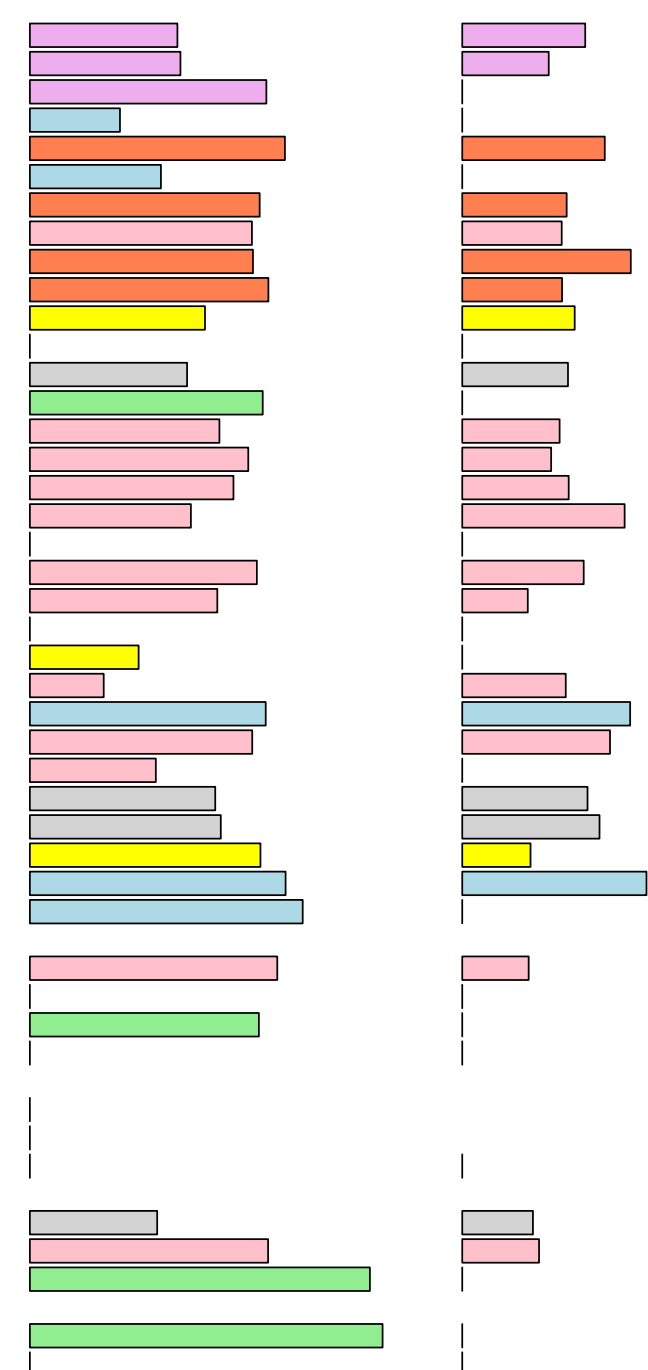

Soil_LWM

Soil_AAN
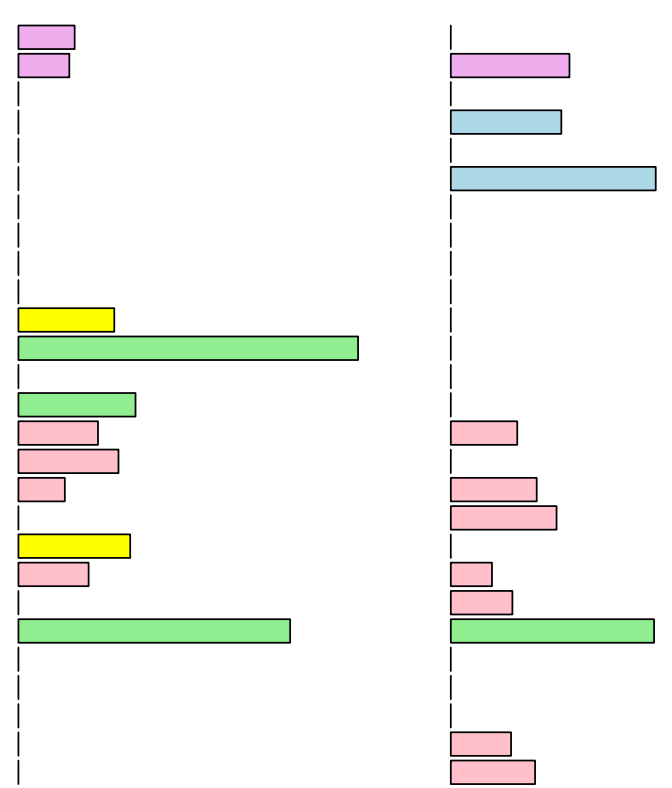
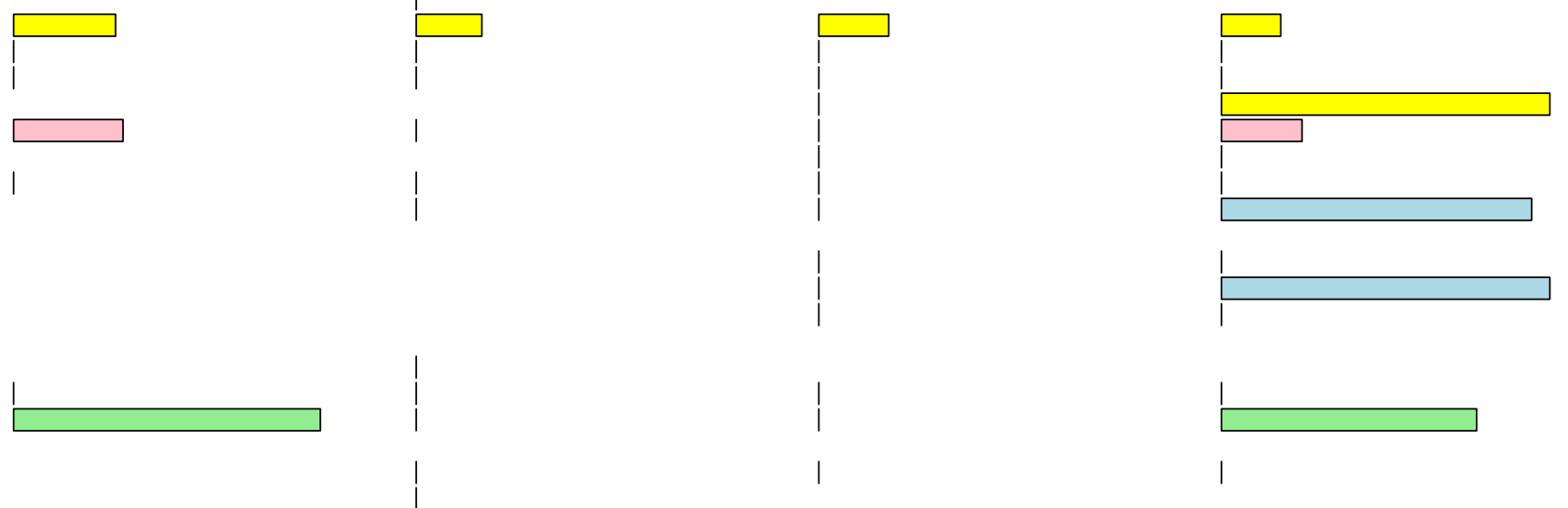

\section{Spearman's rho}

$\square$ Metabolism $\square$ Genetic Information Processing $\square$ Environmental Information Processing $\square$ Cellular Processes $\square$ Organismal Systems $\square$ Human Diseases $\square$ Unclassified 